\title{
Human amniotic epithelial cell feeder layers maintain iPS cell pluripotency by inhibiting endogenous DNA methyltransferase 1
}

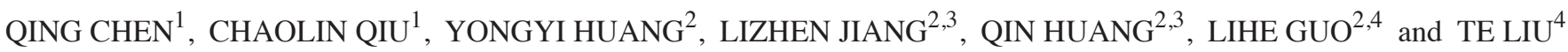 \\ ${ }^{1}$ Shanghai Pudong New Area Gongli Hospital, Shanghai 200135; ${ }^{2}$ Institute of Biochemistry and Cell Biology, Shanghai \\ Institute for Biological Sciences, Chinese Academy of Sciences, Shanghai 200031; \\ ${ }^{3}$ Sino-America United Stem Cell Research Center, Shanghai 200333; ${ }^{4}$ Shanghai Geriatric Institute of Chinese Medicine, \\ Longhua Hospital, Shanghai University of Traditional Chinese Medicine, Shanghai 200031, P.R. China
}

Received July 22, 2013; Accepted August 22, 2013

DOI: $10.3892 /$ etm.2013.1279

\begin{abstract}
Maintaining induced pluripotent stem (iPS) cells in an undifferentiated, self-renewing state during long-term cultivation is, at present, a major challenge. We previously showed that human amniotic epithelial cells (HuAECs) were able to provide a good source of feeder cells for mouse and human embryonic or spermatogonial stem cells; however, the epigenetic mechanisms have not been elucidated. In the present study, mouse embryonic fibroblasts (MEFs) and HuAECs were compared as feeder layers for the long-term culture of human iPS cells. The HuAEC feeders allowed human iPS cells to maintain a high level of alkaline phosphatase (AP) activity and to express key stem cell markers during long-term subculture whereas the MEF feeders did not,. Moreover, the HuAEC feeders significantly affected the cell cycle regulation of the iPS cells, maintaining them in the resting stage and the early stage of DNA synthesis (G0/G1 stage). Furthermore, the $\mathrm{CpG}$ islands of the Nanog and Oct4 promoters were hypomethylated, while the Nanog-and Oct4-specific loci exhibited higher levels of histone $\mathrm{H} 3$ acetylation and lower levels of H3K27 trimethylation in iPS cells cultured on HuAECs compared with those cultured on MEFs. The DNA methyltransferase 1 (DNMT1) expression in iPS cells cultured on HuAECs was shown to be lower than in those cultured on MEFs. In addition, DNMT1-silenced human iPS cells were able to maintain pluripotency over long-term culture on MEFs. In combination,
\end{abstract}

Correspondence to: Dr Te Liu, Room 410, Shanghai Geriatric Institute of Chinese Medicine, Longhua Hospital, Shanghai University of Traditional Chinese Medicine, 558 Xiangyang Road, Shanghai 200031, P.R. China

E-mail: teliu79@126.com; liute1979@126.com; liute79@yahoo.com

Professor Lihe Guo, Institute of Biochemistry and Cell Biology, Shanghai Institute for Biological Sciences, Chinese Academy of Sciences, 320 Yue-yang Road, Shanghai 200031, P.R. China

E-mail: guolihe@cell-star.com.cn

Key words: human amniotic epithelial cells, human induced pluripotent stem cells, DNA methyltransferase 1, pluripotency maintenance these results suggest that endogenous DNMT1 expression in human iPS cells may be regulated by HuAEC feeder cells and that Nanog and Oct4 are crucial components required for the maintenance of iPS cells in an undifferentiated, proliferative state, capable of self-renewal.

\section{Introduction}

Induced pluripotent stem (iPS) cells, generated from murine somatic fibroblast cells by Takahashi and Yamanaka (1), have revolutionized modern science (1-9). The iPS cells represent an appealing option for the derivation of pluripotent patient-specific cells, as no clinically useful embryos or oocytes are employed, which thereby avoids ethical obstacles (10). The original iPS cells were generated by viral transduction of a limited set of transcription factors (Oct3/4, Sox2, c-Myc and Klf4; or Oct3/4, Sox2, Lin28 and Nanog), which reprogrammed somatic cells into pluripotent, embryonic stem cell (ESC)-like cells (10). Since then, iPS cells have been generated in different species by a number of methods $(6,8,11-17)$, sharing with ESCs the key properties of unlimited self-renewal and pluripotency. Although human iPS cells may become a more readily available source of cells for clinical treatment, the question of whether they are able to maintain their cell self-renewal and pluripotency during in vitro culture remains. In our previous studies, we indicated that the expression of numerous growth factors, including basic fibroblast growth factor (bFGF), epidermal growth factor (EGF) and insulin-like growth factor 1 (IGF-1), and leukemia inhibitory factor (LIF) by human amniotic epithelial cells (HuAECs) may be crucial for the function of feeder cells in maintaining mouse and human ESCs, as well as mouse spermatogonial stem cells, in an undifferentiated, proliferative state, capable of self-renewal (18-21). Furthermore, we have demonstrated that HuAEC-dependent epigenetic modifications of the $c-M y c$ gene locus occur in the previously mentioned stem cells, providing a possible mechanism for their HuAEC-dependent maintenance in an undifferentiated state (18-20). Although we previously demonstrated that HuAECs were able to be effectively used as feeder cells, very little is known about how they maintain iPS cell self-renewal and inhibit the differentiation of the iPS cells. 
In a previous study, Nanog and Oct4 were shown to be two key factors required to maintain the pluripotency of ESCs, iPS cells and early embryos; they are co-expressed in developmental stage- and cell type-specific manners (22). The Nanog gene is expressed in pluripotent cells, including ESCs, embryonic carcinoma and embryonic germ cells, and its transcripts are present in the interior cells of the compacted morula and the inner cell mass of the blastocyst (22). Oct4 is also necessary for maintaining the pluripotency of cells of inner cell mass lineage (22), and its expression has also been observed in ESCs and iPS cells. The reduction in Oct4 expression leads to trans-differentiation of ESCs into trophoblast stem cells under adequate culture conditions (22). Previous studies have proposed that partial DNA demethylation in restricted areas in the Oct4 regulatory region is required for gene activation (3,23-26). The Nanog promoter is also demethylated in nuclear transfer ESCs, fibroblast ESCs and in transduced cells $(3,23,27)$. Moreover, DNA methyltransferase (DNMT)-1 and DNMT3 (a/b) have been shown to contribute synergistically to the methylation of Oct4 and Nanog during mouse embryonic cell differentiation in vivo (28).

Epigenetic regulation, particularly DNA methylation, is crucial in gene silencing in mammals (28). DNA methylation is important for establishing the dynamic chromatin configuration of the genome in pluripotent ESCs and iPS cells and for coordinating genomic reorganization during cell differentiation (29). A number of key proteins have been shown to affect epigenetic modifications via DNA methylation, most importantly the DNA methyltransferases, DNMT1, DNMT3a and DNMT3b (30). DNMT1 is the 'maintenance methyltransferase' that localizes to replication foci during the $S$ phase and copies the DNA methylation pattern to the newly synthesized daughter strand (31,32). DNMT3a and DNMT3b are de novo methyltransferases, responsible for the methylation of unmodified DNA $(31,32)$. Sen et al $(33)$ have indicated that the DNMT1 protein is predominantly confined to cells of the basal layer of adult human epidermal tissue and is absent from the outer differentiated layer. Therefore, DNMT1 is expressed in epidermal progenitor-containing cell populations and is lost during differentiation (33). However, a DNMT1, DNMT3a and DNMT3b triple-knockout ESC line was shown to grow robustly and maintain its undifferentiated characteristics (29). In addition, when ESCs or iPS cells are treated with 5-aza-cytidine (a DNA methyltransferase inhibitor), the influence of DNMT1 is weakened and DNA hypomethylation occurs during cell reprogramming (34). Although DNMT1 is frequently designated as a maintenance methyltransferase, while DNMT3a and DNMT3b are classified as de novo methyltransferases, these enzymes have been shown to exhibit overlapping functions (29). Moreover, in spite of a 5-to-30-fold higher preference of DNMT1 for hemimethylated DNA, it exhibits greater de novo DNA methyltransferase activity in vitro and is present at higher levels than DNMT3a and DNMT3b in ESCs and somatic cells (35).

Experimentally, human iPS cells are highly similar to human ESCs in terms of morphology, proliferation, gene expression and the epigenetic status of pluripotency-specific genes (21). Furthermore, the global epigenetic landscapes, as indicated by the distribution of histone modifications and DNA methylation, are very similar between ESCs and iPS cells (29).
Therefore, the cells employ the same molecular mechanisms to maintain the expression of the pluripotency regulators Nanog and Oct4 and to maintain their properties via epigenetic modifications (36). Our preliminary experiments revealed that HuAECs were able to be effectively used as feeder cells to maintain iPS cell self-renewal and inhibit iPS cell differentiation. iPS cells simultaneously express high levels of Oct4 and Nanog when cultured on HuAECs. Accordingly, we hypothesized that the low endogenous activity of DNMT1, DNMT3a and/or DNMT3b in human iPS cells may lead to hypomethylation of the $\mathrm{CpG}$ islands on the promoter regions of Nanog and Oct4 and that the high expression of these factors, modulated by HuAECs feeder layers, may maintain the pluripotency and self-renewal properties of the iPS cells.

\section{Materials and methods}

Preparation of mouse embryonic fibroblasts (MEFs) and HuAECs. MEF cells were isolated from 13-day-old C57BL/6 mouse embryos. Cells were mitotically inactivated using mitomycin C (Sigma-Aldrich, St. Louis, MO, USA), as described previously (18). The mitotic inactivation of MEF cells was conducted by treatment with $10 \mu \mathrm{g} / \mathrm{ml}$ mitomycin $\mathrm{C}$ for $2 \mathrm{~h}$ at $37^{\circ} \mathrm{C}$. The cells were washed three times with phosphate-buffered saline (PBS), digested with $0.25 \%$ trypsin-EDTA solution (cat no. 25300-054; Invitrogen Life Technologies, Carlsbad, CA, USA) and plated at a density of $1 \times 10^{5} / \mathrm{ml}$, with $2.5 \mathrm{ml}$ in each well of a gelatin-coated six-well dish. Human placentas were obtained with written and informed consent from pregnant females who were negative for human immunodeficiency virus (HIV)-I, hepatitis B and hepatitis C. The study received approval for the appropriate use of human amnion by the institutional Ethics Committee of Shanghai Geriatric Institute of Chinese Medicine (Shanghai, China). Amniotic membranes were mechanically separated from the chorions of placentas, which were obtained from females who had undergone an uncomplicated Cesarean section. HuAECs were harvested from the epithelial layers (with the basement membrane attached) of the obtained amniotic membranes, as described in a previous study, with certain modifications (18). In brief, the membrane was placed in a 250 -ml flask containing Dulbecco's modified Eagle's medium (DMEM) and cut with a razor to produce $0.5-1.0 \mathrm{~cm}^{2}$ segments. The segments were subsequently digested with $0.25 \%$ trypsin-EDTA at $37^{\circ} \mathrm{C}$ for $45 \mathrm{~min}$ and the resulting cell suspensions were seeded in a six-well plate in DMEM supplemented with $10 \%$ fetal calf serum (FCS; PAA Laboratories GmbH, Pasching, Austria), penicillin $(100 \mathrm{U} / \mathrm{ml})$ and glutamine $(0.3 \mathrm{mg} / \mathrm{ml})$. Following this, the cells were incubated in a humidified tissue culture incubator containing $5 \% \mathrm{CO}_{2}$ at $37^{\circ} \mathrm{C}$. The $\mathrm{HuAECs}$ were grown to a density of $\sim 100 \%$ and were subsequently used as feeder layers for human iPS cell culture following mitomycin C (Sigma-Aldrich) treatment.

Co-culture of human iPS cells with HuAECs and MEFs. The human iPS cells were generated by our laboratory and derived from $\mathrm{CD}_{3} 4^{+}$human amniotic fluid cells (HuAFCs) via transduction with lentiviral constructs encoding only Oct4, as previously described (37). iPS cultures were separated from the feeder cells by treatment with $0.125 \%$ trypsin-EDTA solution 
and plated onto and co-cultured with HuAECs or MEFs. The cells were cultured in DMEM:F12 (1:1) medium supplemented with $15 \%$ KnockOut $^{\text {TM }}$ Serum Replacement (Invitrogen Life Technologies), $1 \mathrm{mM}$ sodium pyruvate, $2 \mathrm{mM}$ L-glutamine, $0.1 \mathrm{mM}$ nonessential amino acids, $0.1 \mathrm{mM} \beta$-mercaptoethanol, penicillin $(25 \mathrm{U} / \mathrm{ml})$-streptomycin $(925 \mathrm{mg} / \mathrm{ml})$, and mixed, without LIF. The cells were incubated in a humidified tissue culture incubator containing $5 \% \mathrm{CO}_{2}$ at $37^{\circ} \mathrm{C}$. All cells were cultured on the same feeder until the 10 th passage, prior to being used for subsequent experiments.

Alkaline phosphatase (AP) staining. The AP activity of human iPS cells, which were cultured on HuAECs or MEFs, was determined using an alkaline phosphatase detection kit (Sigma-Aldrich), in accordance with the manufacturer's instructions (38).

RNA extraction and analysis using quantitative polymerase chain reaction $(q P C R)$. Total-RNA from each cell was isolated using TRIzol reagent ${ }^{\circledR}$ (Invitrogen Life Technologies), in accordance with the manufacturer's instructions. The RNA samples were treated with DNase I (Sigma-Aldrich), quantified and reverse-transcribed into complementary DNA (cDNA) with the ReverTra Ace- $\alpha$ First Strand cDNA Synthesis kit [Toyobo (Shanghai) Biotech Co., Ltd., Shanghai, China]. qPCR was conducted with a RealPlex4 real-time PCR detection system from Eppendorf (Hamburg, Germany), with SYBR ${ }^{\circledR}$ Green Real-Time PCR Master Mix [Toyobo (Shanghai) Biotech Co., Ltd.] as the detection dye. The qPCR amplification was performed over 40 cycles with denaturation at $95^{\circ} \mathrm{C}$ for $15 \mathrm{sec}$ and annealing at $58^{\circ} \mathrm{C}$ for $45 \mathrm{sec}$. The target cDNA was quantified using the relative quantification method. A comparative threshold cycle $(\mathrm{Ct})$ was used to determine gene expression relative to a control (calibrator); steady-state mRNA levels are expressed as an $n$-fold difference relative to the calibrator. For each sample, the maker gene $\mathrm{Ct}$ values were normalized with the formula: $\Delta \mathrm{Ct}=\mathrm{Ct}$ _genes - $\mathrm{Ct}$-18S RNA. To evaluate the relative expression levels, the following formula was used: $\Delta \Delta \mathrm{Ct}=\Delta \mathrm{Ct}$ _all_groups $-\Delta \mathrm{Ct}$ _blank_control_group. The values used to the plot relative expression of the markers were calculated using the expression $2^{-\Delta \Delta C t}$ method. The mRNA levels were calibrated on the basis of levels of $18 \mathrm{~S}$ ribosomal RNA (rRNA). The cDNA of each gene was amplified with primers as previously described $(21,37,39,40)$.

RNA interference and transfection. The small interfering RNA (siRNA)-DNMT1 plasmid was manufactured by Shanghai GenePharma, Ltd. (Shanghai, China) and the methods used for plasmid transfection were in accordance with the company's instructions. In brief, iPS cells were cotransfected with $0.3 \mu \mathrm{g}$ siRNA-DNMT1 expression plasmid or siRNA-Mock plasmid, respectively, using Lipofectamine 2000 reagent (Invitrogen, Life Technologies Corporation, Grand Island, NY, USA), in accordance with the manufacturer's instructions. The cells were seeded in a six-well plate and cultured in DMEM:F12 (1:1) medium supplemented with $15 \%$ KnockOut $^{\mathrm{TM}}$ Serum Replacement, $1 \mathrm{mM}$ sodium pyruvate, $2 \mathrm{mM}$ L-glutamine, $0.1 \mathrm{mM}$ nonessential amino acids, $0.1 \mathrm{mM} \beta$-mercaptoethanol, penicillin
(25 U/ml)-streptomycin $(925 \mathrm{mg} / \mathrm{ml})$ and mixed, without LIF. The cells were incubated in a humidified tissue culture incubator containing $5 \% \mathrm{CO}_{2}$ at $37^{\circ} \mathrm{C}$ until $80 \%$ confluence was achieved.

Flow cytometric (FCM) analysis of cell cycle by propidium iodide $(P I)$ staining. Each group of cells was seeded at a density of $3 \times 10^{5}$ cells per well in six-well plates and cultured until $85 \%$ confluent. Following this, each group of cells was washed three times with PBS, prior to being subjected to centrifugation (Allegra X-22 ${ }^{\circledR}$; Beckman Coulter, Miami, FL, USA) at $1,000 \mathrm{x} \mathrm{g}$ for $5 \mathrm{~min}$. The cell pellets were then resuspended in $1 \mathrm{ml} \mathrm{PBS}$, fixed in $70 \%$ ice-cold ethanol and stored in a freezer for $>48 \mathrm{~h}$. Prior to FCM analysis, the fixed cells were centrifuged, washed twice with PBS and resuspended in PI staining solution (Sigma-Aldrich) containing $50 \mu \mathrm{l} / \mathrm{ml} \mathrm{PI}$ and $250 \mu \mathrm{g} / \mathrm{ml}$ RNase A (Sigma-Aldrich). The cell suspensions, which were hidden from light, were incubated for $30 \mathrm{~min}$ at $4^{\circ} \mathrm{C}$ and analyzed using a fluorescence-activated cell sorter (FACS; FCM-500; Beckman Coulter). A total of 20,000 events were acquired for analysis using CellQuest software (BD Biosciences, Franklin Lakes, NJ, USA).

Bisulfite conversion of genomic DNA and methylation-specific PCR (MS-PCR). The cells were lysed in DNA lysis buffer [0.5\% sodium dodecyl sulfate (SDS), 0.1 M EDTA, $10 \mathrm{mM}$ Tris- $\mathrm{HCl}(\mathrm{pH} 8.0)$ and $100 \mathrm{ng} / \mathrm{ml}$ proteinase $\mathrm{K}$; all from Sigma-Aldrich] and incubated at $55^{\circ} \mathrm{C}$ for $2 \mathrm{~h}$. The treatment of genomic DNA and the MS-PCR assay were performed as previously described $(2,5,9,18,37)$. In addition, the specific primers for Nanog and Oct-4 were designed as previously described $(2,5,9,18,37)$. The PCR products were separated using $12 \mathrm{~g} / 1$ ethidium bromide containing agarose gel electrophoresis with $1 \mathrm{X}$ Tris acetate EDTA (TAE) buffer, and visualized under UV illumination.

Chromatin immunoprecipitation (ChIP) assays. ChIP experiments were conducted using anti-acetylated histone $\mathrm{H} 3$ antibody (Upstate Biotechnology, Inc., Lake Placid, NY, USA), anti-trimethylated H3K27 antibody (Abcam, Cambridge, UK) and normal rabbit immunoglobulin G (IgG; Upstate Biotechnology, Inc.) as a negative control. All steps were performed as previously described $(2,5,9,18,37)$. The cells were fixed using $1 \%$ formaldehyde for $30 \mathrm{~min}$ at $37^{\circ} \mathrm{C}$ and then quenched using $125 \mathrm{mM}$ glycine for $10 \mathrm{~min}$ at room temperature to form DNA-protein cross-links. Following this, the samples were placed on ice and sonicated until chromatin fragments became 200-1,000 bp in size. The samples were then incubated with antibodies at $4^{\circ} \mathrm{C}$ overnight. The PCR amplification was performed under the following conditions: 33 cycles of denaturation at $95^{\circ} \mathrm{C}$ for $30 \mathrm{sec}$, annealing at $55^{\circ} \mathrm{C}$ for $30 \mathrm{sec}$ and extension at $72^{\circ} \mathrm{C}$ for $30 \mathrm{sec}$.

Immunofluorescence (IF) staining. The cultured cells were washed three times with FCS and fixed with $4 \%$ paraformaldehyde (Sigma-Aldrich) for $30 \mathrm{~min}$. The cells were then washed using Tris-buffered saline containing $0.1 \%$ Triton $\mathrm{X}-100$ [TBST-100 buffer; $25 \mathrm{mM}$ Tris- $\mathrm{HCl}$ (pH 8.0), $125 \mathrm{mM}$ $\mathrm{NaCl}$ and $0.1 \%$ Triton $\mathrm{X}-100]$ three times, prior to blocking. Following blocking, the cells were incubated with rabbit 

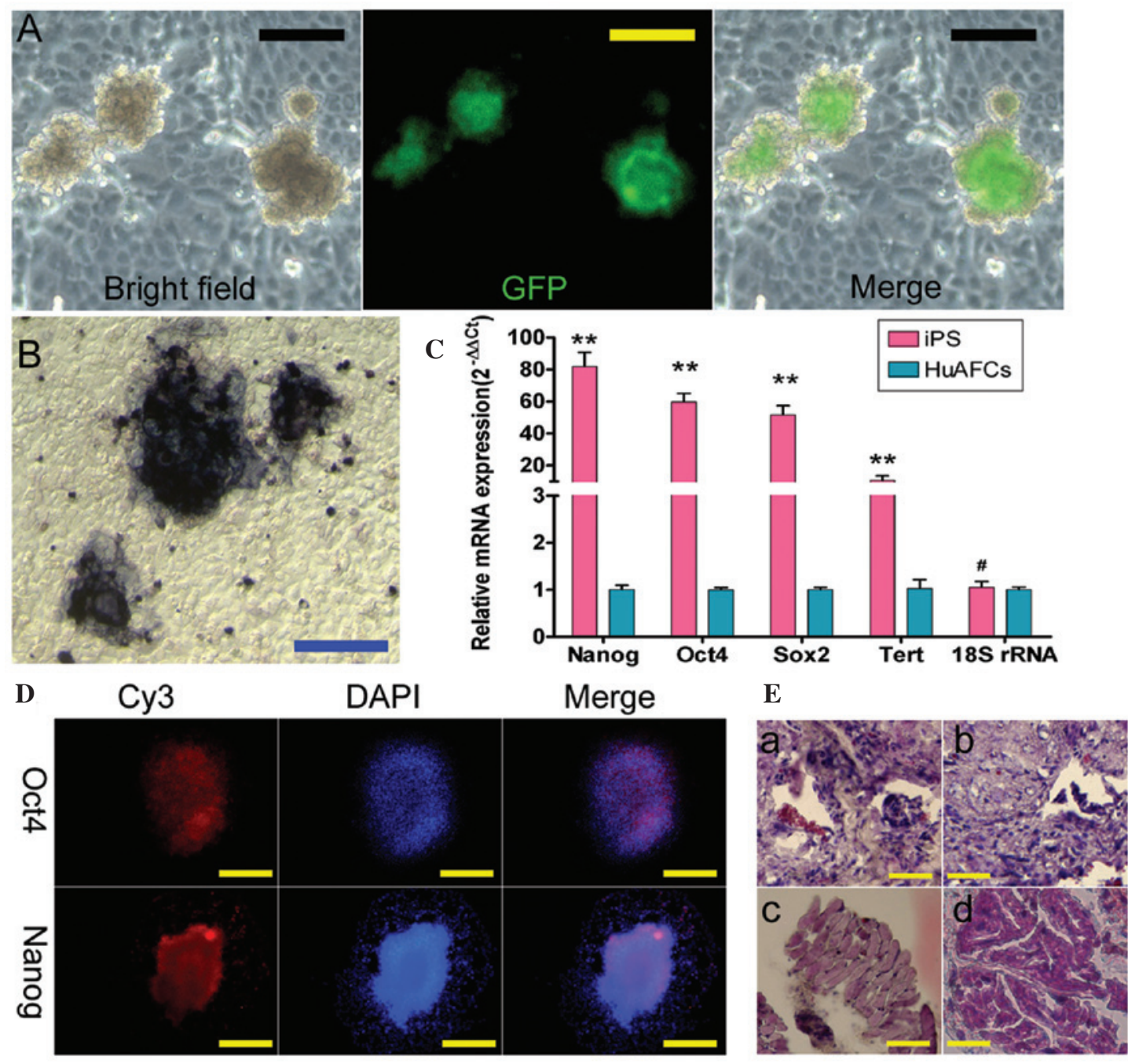

Figure 1. Characterization of reprogrammed human induced pluripotent stem (iPS) cells. (A) Bright-field image, green fluorescent protein (GFP)-positive clones and merged GFP and bright-field images of human iPS cells in the seventh phase of culture on human amniotic epithelial cell (HuAEC) feeder layers. Scale bar $=50 \mu \mathrm{m}$. (B) Deep blue staining for alkaline phosphatase (AP) activity in a human iPS cell colony. The AP activity in human iPS cells cultured on HuAECs was steady in the seventh phase. Scale bar=50 $\mu \mathrm{m}$. (C) Quantitative reverse transcription-polymerase chain reaction analysis of transcriptional expression of endogenous stem cell markers (Oct4, Nanog, Sox 2 and Tert) in human iPS cells relative to human amniotic fluid cells (HuAFCs) ${ }^{* *} \mathrm{P}<0.01$ vs. HuAFCs; ${ }^{\text {P }}>0.05$ vs. HuAFCs; $\mathrm{n}=3$. (D) Immunostaining was performed on human iPS cells using antibodies against Nanog and $O c t 4$. Scale bar=50 $\mu \mathrm{m}$. DAPI, 4',6-diamidino-2-phenylindole. (E) Histology of teratomas in severe combined immunodeficient mice and histology of a teratoma composed of ectodermal, endodermal and mesodermal tissue. Scale bar=100 $\mu \mathrm{m}$. (a and b) Adipose tissue (mesodermal) and medullary tube (ectodermal), respectively; (c) striated muscle (mesodermal); and (d) gastrula (endodermal).

anti-human Oct3/4 polyclonal antibody (1:200; Chemicon, Temecula, CA, USA) and rabbit anti-human Nanog polyclonal antibody (1:200; Chemicon) overnight at $4^{\circ} \mathrm{C}$, and then with Cy3-conjugated goat anti-rabbit IgG antibody (1:200; Abcam) and $5 \mu \mathrm{g} / \mathrm{ml} \mathrm{4',6-diamidino-2-phenylindole} \mathrm{(DAPI;}$ Sigma-Aldrich) at room temperature for $30 \mathrm{~min}$. Following this, the cells were thoroughly washed with TBST-100 and viewed under a fluorescence microscope (DMI3000; Leica Camera Inc., Allendale, NJ, USA).

Western blot analysis. Cells were lysed using a $2 \mathrm{X}$ loading lysis buffer [50 mM Tris- $\mathrm{HCl}$ ( $\mathrm{pH} 6.8$ ), 2\% sodium dodecyl sulfate, $10 \% \beta$-mercaptoethanol, $10 \%$ glycerol and $0.002 \%$ bromphenol blue]. The total quantity of proteins from the cultured cells was subjected to $12 \%$ SDS-polyacrylamide gel electrophoresis (PAGE) and transferred onto Hybrid-polyvinylidene fluoride (PVDF) membranes (Millipore, Bedford, MA, USA).
Following blocking with $5 \%(\mathrm{w} / \mathrm{v})$ non-fat dried milk in Tris-buffered saline containing Tween-20 [TBST-20; $25 \mathrm{mM}$ Tris- $\mathrm{HCl}$ (pH 8.0), $125 \mathrm{mM} \mathrm{NaCl}$ and $0.05 \%$ Tween-20], the PVDF membranes were washed four times (15 min each) with TBST-20 at room temperature and incubated with primary antibody. Following extensive washing, the membranes were incubated with horseradish peroxidase (HRP)-conjugated goat anti-rabbit IgG secondary antibody (1:1,000; Santa Cruz Biotechnology, Inc., Santa Cruz, CA, USA) for $1 \mathrm{~h}$. The membranes were then washed four times (15 min each) with TBST-20 at room temperature, prior to the immunoreactivity being visualized by enhanced chemiluminescence (ECL) using an ECL Chemiluminescent Substrate Reagent kit from Perkin-Elmer Life Science (Norwalk, CT, USA).

Teratoma formation. All animal procedures were conducted at Shanghai University of Traditional Chinese Medicine 

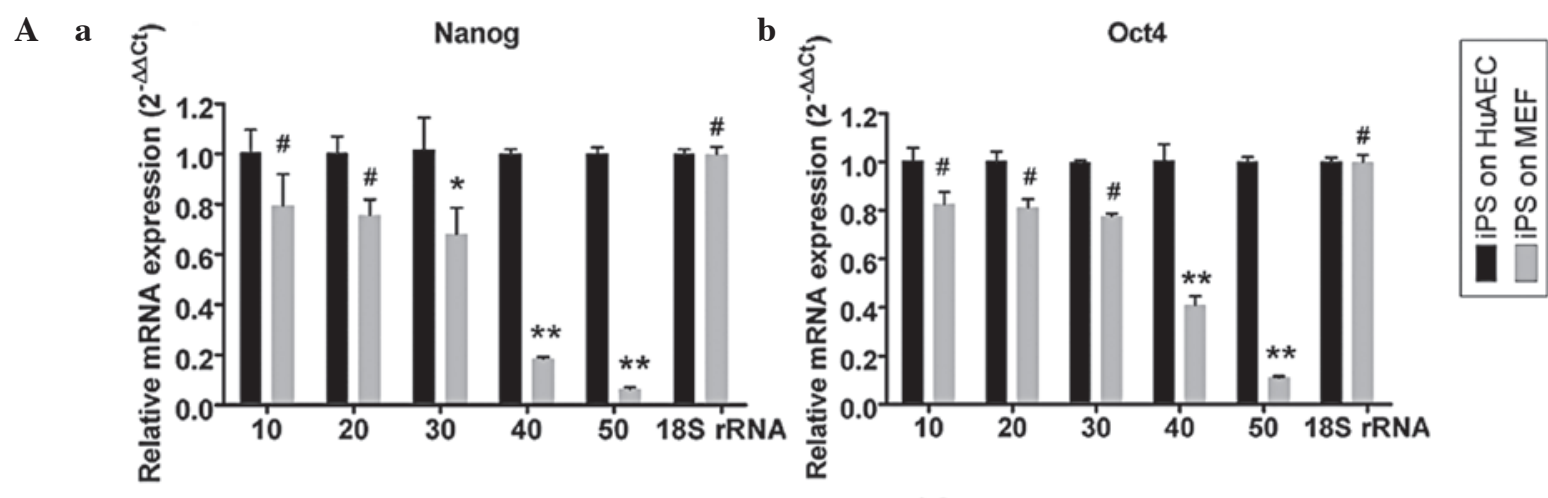

B a

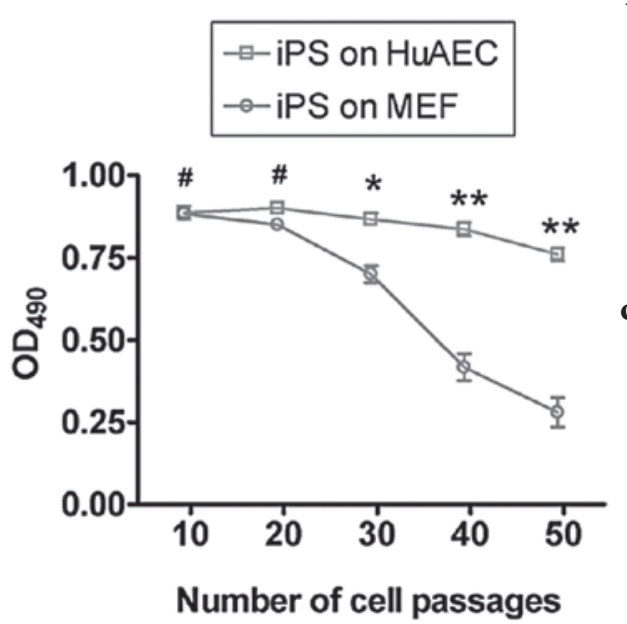

b

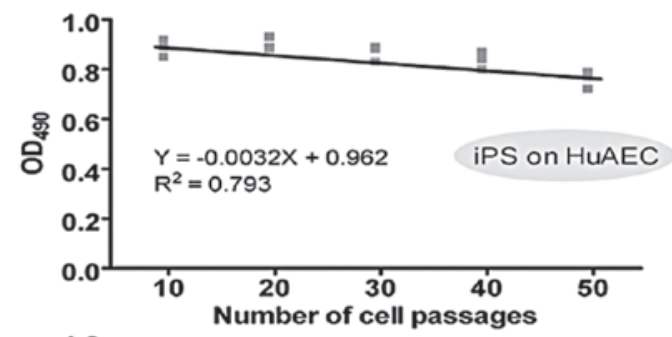

c

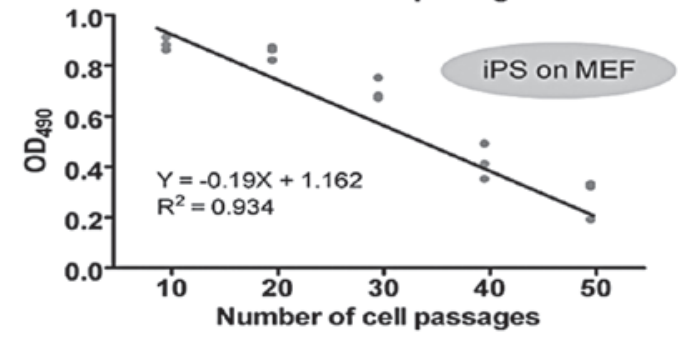

Figure 2. Expression of Nanog and Oct4 and alkaline phosphatase (AP) activity in human induced pluripotent stem (iPS) cells at different passages. (A) Quantitative reverse transcription-polymerase chain reaction analysis showed that the levels of (a) Nanog and (b) Oct4 expressed in the iPS cells cultured on mouse embryonic fibroblasts (MEFs) reduced rapidly, while those of iPS cells cultured on human amniotic epithelial cells (HuAECs) did not significantly change. Relative mRNA expression is shown following normalization to $18 \mathrm{~S}$ ribosomal RNA (rRNA), serving as an internal control. "P<0.05 vs. iPS cells on HuAECs; ${ }^{* *} \mathrm{P}<0.01$ vs. iPS cells on HuAECs; ${ }^{*} \mathrm{P}>0.05$ vs. iPS cells on HuAECs; $\mathrm{n}=3$. (Ba) AP activity assay of human iPS cells cultured on HuAECs or MEFs. The AP activity of human iPS cells cultured on MEFs (c) was decreased compared with those cultured on HuAECs (b) from the 30th to 50th passage; ${ }^{*}<<0.05$ vs. iPS cells on MEFs; ${ }^{* *} \mathrm{P}<0.01$ vs. iPS cells on MEFs; ${ }^{*} \mathrm{P}>0.05$ vs. iPS cells on MEFs; $n=3$. OD, optical density.

(Shanghai, China) with approval from the Institutional Animal Care and Use Committee and in accordance with the institutional guidelines. Human iPS cells $\left(1 \times 10^{6}\right)$ were inoculated into the hind legs of severe combined immunodeficient (SCID) mice. Teratomas were embedded in paraffin and histologically examined following hematoxylin and eosin staining. The procedure for the teratoma formation experiment was performed as described previously (18).

Statistical analysis. Each experiment was performed as least three times and the data are presented as the mean \pm standard error (SE). The differences were evaluated using Student's t-tests. $\mathrm{P}<0.05$ was considered to indicate a statistically significant difference.

\section{Results}

Pluripotency of iPS cells derived from $C D 34^{+} H u A F C s$. We have previously described the successful generation of iPS cells from $\mathrm{CD}^{2} 4^{+} \mathrm{HuAFCs}$ by transduction with lentiviral constructs encoding only Oct4 (37). In this study, the iPS cells were cultured on HuAEC feeder layers until the 10th passage, prior to use in experiments. After testing the effects of different feeder layers, the pluripotency of iPS cells was assayed. Under the microscope, a number of ESC-like colonies were observed among the feeder cells; these Oct4-green fluorescent protein (GFP)-positive colonies appeared isolated and rounded, consistent with more undifferentiated cells (Fig. 1A). Furthermore, the AP activity of these human iPS cells was high, as represented by the deep blue staining on the surface of the colonies (Fig. 1B). In addition, the IF staining revealed that the expression levels of the pluripotent stem cell markers, Nanog, Oct4 and Sox2, were increased in the iPS colonies (Fig. 1D). Consistent with the IF results, qPCR analysis showed that the expression levels of these stem cell markers were $\sim 60-100$-fold higher in human iPS cells than in HuAFCs, which served as an internal control (Fig. 1C). Moreover, the high level of telomerase activity in human iPS cells suggested that their replicative life-span was likely to exceed that of somatic cells. Meanwhile, in the in vivo xenograft experiments, teratomas formed on the legs of SCID mice injected with the iPS cells (Fig. 1E). The iPS-derived teratomas contained cellular representatives of all three germ layers (Fig. 1E). Based on these results, it was concluded that the human iPS cells derived from HuAFCs possessed strong pluripotency. 
$\mathbf{A}$

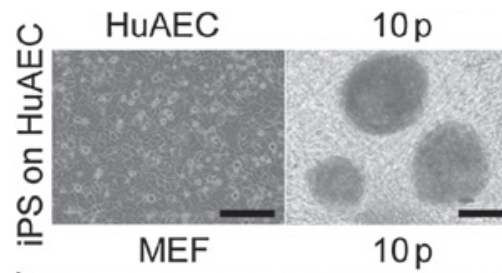

$20 p$

$30 \mathrm{p}$

$40 p$

$50 p$

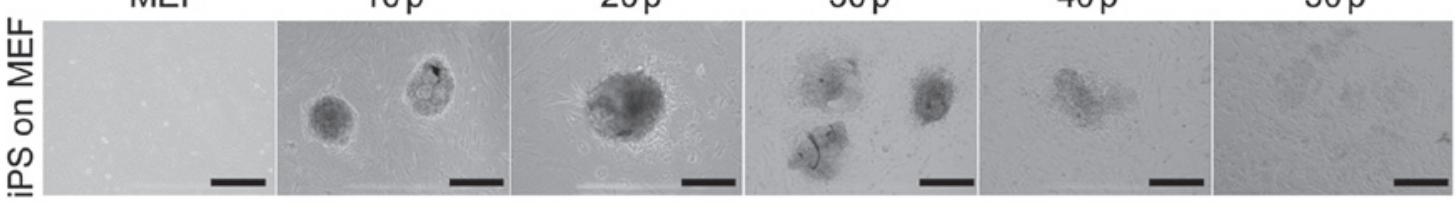

B

$10 p$

$30 \mathrm{p}$

$50 p$
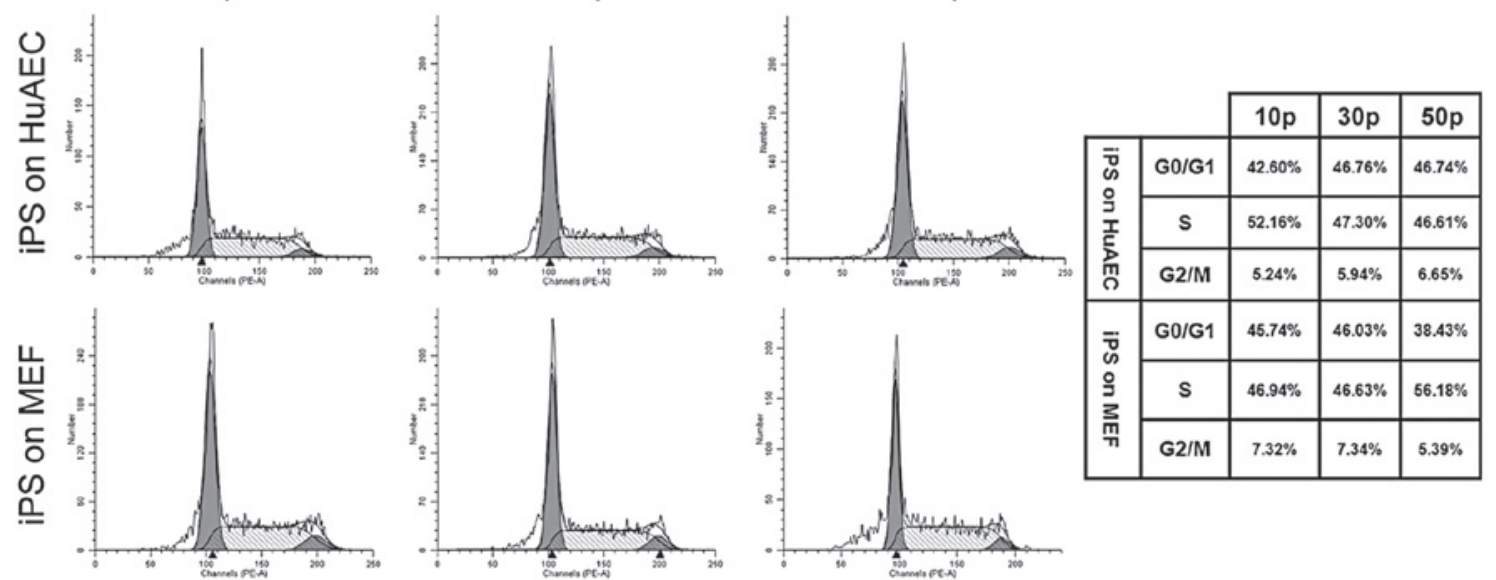

Figure 3. Morphology and cell cycles of human induced pluripotent stem (iPS) cells cultured on different feeder layers during subculture. (A) Morphology of iPS cells on human amniotic epithelial cells (HuAECs) or mouse embryonic fibroblasts (MEFs). Scale bar=50 $\mu$ m. On HuAECs, iPS colonies appeared more isolated and rounded with obvious boundaries with feeder cells, consistent with the appearance of undifferentiated cells during continuous culture. However, on MEFs, iPS colonies appeared to be migrating into the feeder layer with a less distinct cellular boundary, consistent with the appearance of more differentiated cells from the 40th passage. (B) The cell cycle of iPS cells cultured on MEFs or HuAECs was tested using flow cytometric analysis. The cell cycles of the iPS cells cultured on HuAECs were not markedly different between the 10th, 30th and 50th passages, indicating that long-term culture on HuAEC feeder layers did not affect the process of cell division in the iPS cells. Moreover, HuAEC feeder layers were able to maintain human iPS cells in the resting stage and early stage of DNA synthesis (G0/G1 stage). p, passage.

Pluripotency of iPS cells cultured on MEFs is weakened during successive subcultures in vitro. iPS cells were successively subcultured either on HuAECs or MEFs in order to evaluate the effect of the different feeder layers on pluripotency. The two groups of cells were cultured in uniform conditions and were subcultured in succession until passage 50. Since AP levels decrease as stem cells lose their pluripotency and differentiate, the AP activity of human iPS cells cultured on different feeder layers was analyzed at every 10th passage. The results showed that the AP activity of the iPS cells cultured on MEFs reduced rapidly and there was a significant negative correlation between the AP activity level and the number of cell passages $\left(\mathrm{R}^{2}=0.934, \mathrm{P}<0.05\right)$. However, when the iPS cells were cultured on HuAECs, their AP activity level was not significantly altered $\left(\mathrm{R}^{2}=0.793, \mathrm{P}>0.05\right.$; Fig. $\left.2 \mathrm{~B}\right)$. In addition, at the 40th and 50th passage, the AP activity levels of human iPS cells cultured on MEFs $(0.417 \pm 0.041$ and $0.280 \pm 0.045$, respectively) were significantly lower than those on HuAECs $(0.837 \pm 0.021$ and $0.760 \pm 0.022$, respectively). To evaluate whether the pluripotency of iPS cells changed, stem cells markers were assayed using qPCR. The results of the qPCR analysis showed that the Nanog and Oct4 expression levels in iPS cells cultured on MEFs reduced rapidly, while those in iPS cells cultured on HuAECs did not markedly change over time (Fig. 2A).
HUAEC and MEF feeders maintain iPS cells in the resting stage and early stage of DNA synthesis (G0/G1 stage). In the early passages (comparing passages 10, 30 and 50), the iPS cells grown on HuAECs and MEFs exhibited similar cell cycle distributions (Fig. 3). Furthermore, the cell cycles of the iPS cells cultured on HuAECs were not markedly different between the 10th, 30th and 50th passages, indicating that long-term culture on HuAECs feeder layers did not affect the process of cell division in the iPS cells. Similarly, when the iPS cells were cultured on MEFs, the FCM analysis showed no significant differences in the cell cycle distribution at each passage. The iPS cells cultured on MEFs were always in the resting stage and early stage of DNA synthesis (G0/G1 stage; Fig. 3). No significant differences were observed between the cell cycles of the iPS cells cultured on HuAECs or on MEFs at the 10 th, 30 th or 50 th passages.

Changes in DNA and histone epigenetic modifications in iPS cells during consecutive subcultures. The DNA methylation status of the CpG islands in the Nanog and Oct4 promoters was investigated using sodium bisulfite treatment of the iPS cells cultured on HuAECs or MEFs at every 10th passage. All the CpG islands in the Nanog and Oct4 promoter regions were demethylated with no significant differences from 

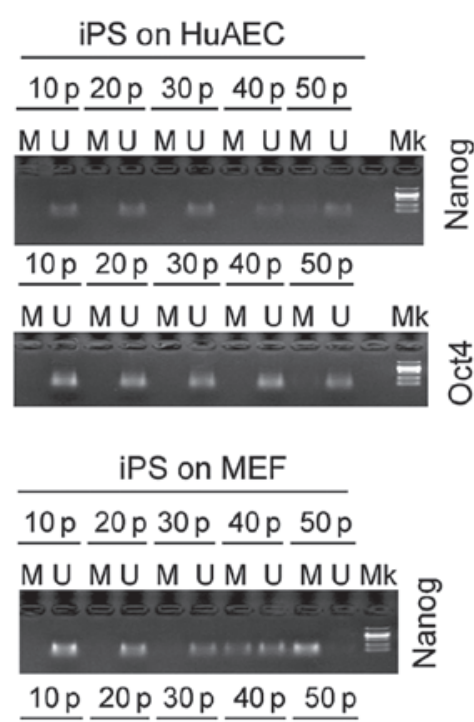

$M U M U M U M U M U M k$

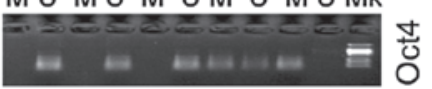

B

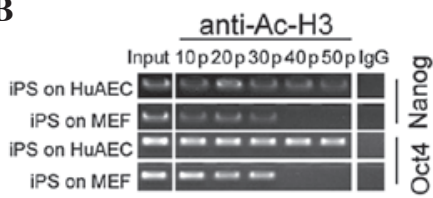

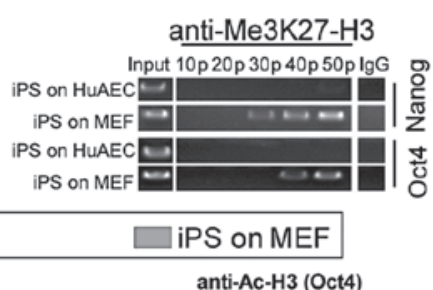

anti-Ac-H3 (Oct4)

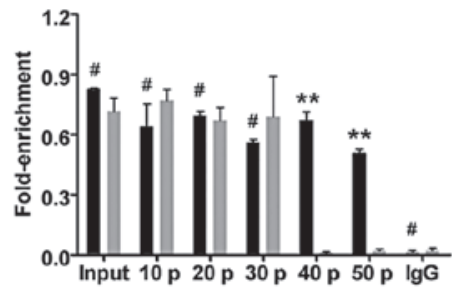

anti-Me3K27-H3 (Oct4)

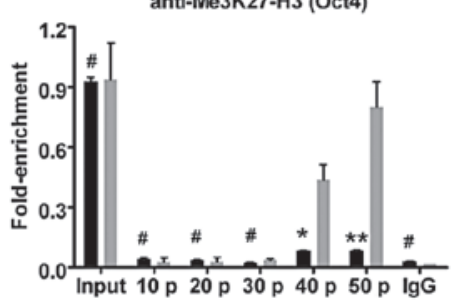

C
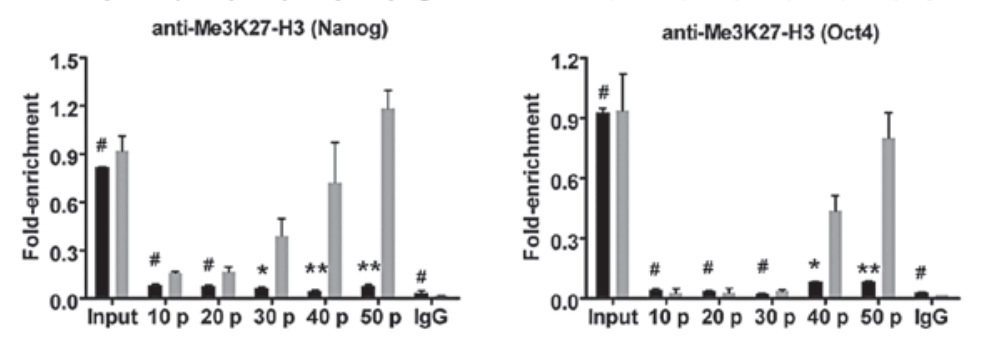

$\begin{array}{lr}\text { iPS on HuAEC } & \text { iPS on MEF } \\ \text { DNMT1 } & 10 p 30 p 50 p\end{array}$

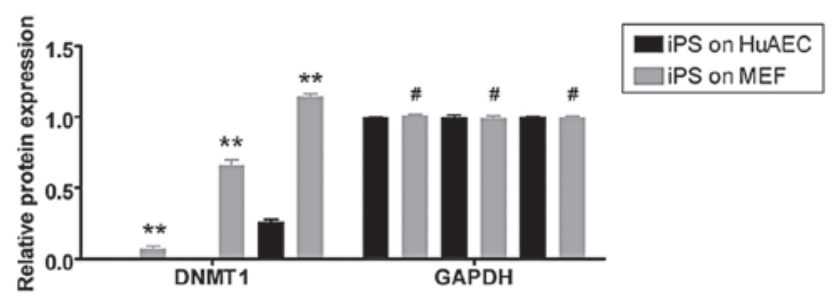

Figure 4. Epigenetic modifications of DNA and histones in human induced pluripotent stem (iPS) cells. (A) Analysis of the methylation state of the CpG islands of the Nanog and Oct4 promoters using sodium bisulfite polymerase chain reaction (PCR) and sequencing. No significant differences in the DNA methylation status of the CpG islands of the Nanog and Oct4 promoters were observed in human iPS cells cultured on human amniotic epithelial cells (HuAECs) compared with those cultured on mouse embryonic fibroblasts (MEFs) from the 10th to 30th passage. However, from the 40th passage, these regions were markedly hypermethylated in the iPS cells cultured on MEFs, although not on HuAECs. M, methylated CpG; U, unmethylated CpG; Mk, DNA marker. (B) Histone H3 acetylation and H3K27 trimethylation of the Nanog and Oct4 loci. PCR-amplified genomic DNA obtained following immunoprecipitation with anti-acetylated histone $\mathrm{H} 3$ (anti-Ac-H3) antibody or anti-trimethylated histone H3K27 (anti-Me3K27-H3) antibody. " $\mathrm{P}<0.05$ vs. iPS cells on MEFs; ${ }^{* *} \mathrm{P}<0.01$ vs. iPS cells on MEFs; "P $>0.05$ vs. iPS cells on MEFs; $n=3$. (C) Western blot analysis showing that the expression of endogenous DNA methyltransferase 1 (DNMT1) in human iPS cells cultured on MEFs was significantly higher than that in iPS cells cultured on HuAECs following the 40th passage. ${ }^{* * *} \mathrm{P}<0.01$ vs. iPS cells on HuAECs; ${ }^{\#} \mathrm{P}>0.05$ vs. iPS cells on HuAECs; n=3.p, passage; GAPDH, glyceraldehyde 3-phosphate dehydrogenase.

the 10th to the 50th passage in human iPS cells cultured on HuAECs (Fig. 4A). However, in iPS cells cultured on MEFs, these regions of the Nanog and Oct4 promoters were moderately demethylated at passages 10, 20 and 30 (Fig. 4A), prior to becoming hypermethylated at passages 40 and 50 . These differences suggested that HuAECs positively regulated the Nanog and Oct4 genes in human iPS cells by maintaining the hypomethylation of promoter $\mathrm{CpG}$ islands. In addition, ChIP assays were performed to evaluate the histone $\mathrm{H} 3$ acetylation levels of the Nanog and Oct4 promoters in human iPS cells cultured on HuAECs or MEFs. In iPS cells cultured on HuAECs, the acetylation and K27 trimethylation of histone $\mathrm{H} 3$ in the Nanog and Oct4 promoters and the 5'untranslated regions (5'UTRs) were similar to those in human iPS cells cultured on MEFs within 20 passages (Fig. 4B). However, from passage 30, histone $\mathrm{H} 3$ appeared relatively hyperacetylated and histone K27 appeared hypomethylated in the Nanog-and Oct4-specific sites in human iPS cells cultured on HuAECs, compared with the same regions in cells cultured on MEFs (Fig. 4B). These results suggested that HuAECs were capable of maintaining the Nanog and Oct4 loci in an active transcriptional state through covalent histone modifications over long-term culture. Western blotting was also used to assess the relative protein expression of DNMT1 in iPS cells cultured on different feeder layers. Over multiple passages, DNMT1 protein was more highly expressed in iPS cells cultured on MEFs than in iPS cells cultured on HuAECs (Fig. 4C). At passages 30 and 50, the DNMT1 protein levels in iPS cells cultured on MEFs were $0.676 \pm 0.040$ and $1.220 \pm 0.021$ relative to glyceraldehyde 3-phosphate dehydrogenase (GAPDH) expression levels, respectively. These values were significantly higher than those in iPS cells cultured on HuAEC feeders $(0.003 \pm 0.001$ and $0.021 \pm 0.010$ relative to GAPDH levels, respectively).

Suppression of endogenous DNMT1 expression in iPS cells maintains their pluripotency while cultured on MEFs. In order to evaluate whether endogenous DNMT1 expression promoted DNA methylation of Nanog and Oct4 and the differentiation 


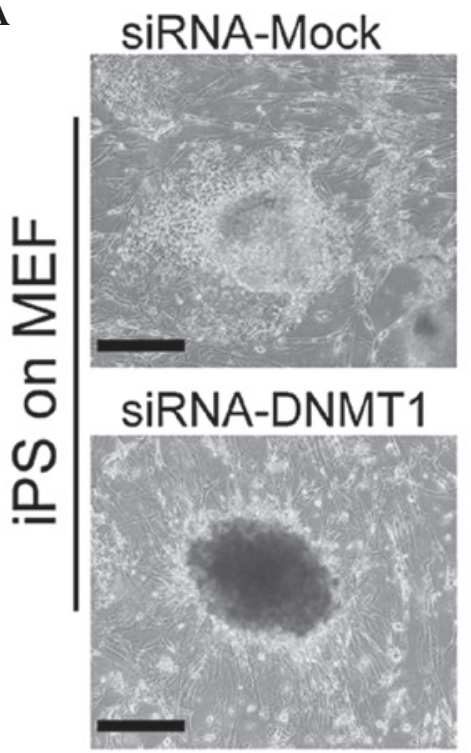

B

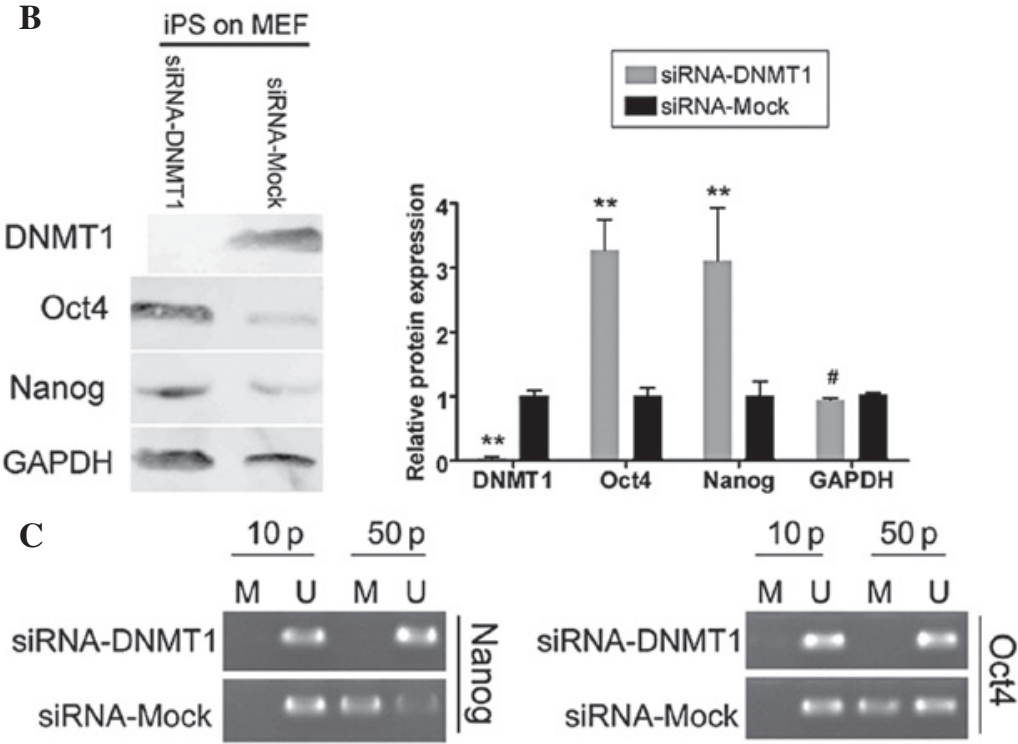

Figure 5. Suppression of endogenous DNA methyltransferase 1 (DNMT1) expression maintains the pluripotency of induced pluripotent stem (iPS) cells cultured on mouse embryonic fibroblast (MEF) feeder layers. (A) Morphology of small interfering RNA (siRNA)-DNMT1 or siRNA-Mock-transfected human iPS cells cultured on MEFs at the 50th passage (p). Scale bar=50 $\mu \mathrm{m}$. (B) Western blot analysis of the expression of endogenous Oct4, Nanog and DNMT1 in siRNA-DNMT1 or siRNA-Mock-transfected human iPS cells cultured on MEFs at the 50th passage. ${ }^{* *} \mathrm{P}<0.01,{ }^{*} \mathrm{P}>0.05$ vs. siRNA-Mock-transfected cells; $\mathrm{n}=3$. GAPDH, glyceraldehyde 3-phosphate dehydrogenase. (C) Analysis of the methylation state of the CpG islands of the Nanog and Oct4 promoters using sodium bisulfite polymerase chain reaction and sequencing. $\mathrm{M}$, methylated $\mathrm{CpG}$; $\mathrm{U}$, unmethylated $\mathrm{CpG}$.

of cultured iPS cells, siRNA-DNMT1 and siRNA-Mock were transfected into iPS cells. The efficiency of the transfected siRNA on mRNA and protein expression was assessed using qPCR and western blotting, respectively, and these experiments were performed on all cell groups subcultured up to passage 50. As shown in Fig. 5B, western blotting indicated that the DNMT1 protein expression in iPS cells transfected with siRNA-Mock $(1.002 \pm 0.089)$ was higher than that in iPS cells transfected with siRNA-DNMT1 $(0.037 \pm 0.020, \mathrm{P}<0.01$, $\mathrm{n}=3$ ). These results demonstrated that siRNA-DNMT1 specifically interfered with DNMT1 expression in iPS cells. Having confirmed that endogenous DNMT1 expression was suppressed by siRNA, the pluripotent stem cell biomarkers Oct4 and Nanog in iPS cells cultured on MEFs were tested using western blotting. The results revealed that the Oct4 and Nanog protein levels in the siRNA-DNMT1-transfected iPS cells cultured on MEFs were $3.277 \pm 0.475$ and $3.108 \pm 0.719$ of GAPDH expression levels, respectively (Fig. 5B). These values were significantly higher than those in the siRNA-Mock-transfected iPS cells $(1.002 \pm 0.128$ and $1.005 \pm 0.228$ of GAPDH levels, respectively). In addition, the promoter regions of Nanog and Oct4 were observed to be moderately hypomethylated or demethylated in siRNA-DNMT1-transfected iPS cells, while they were moderately hypermethylated in siRNA-Mock-transfected iPS cells (Fig. 5C). These differences suggested that DNMT1 positively regulated the Nanog and Oct4 genes in human iPS cells through the de novo or maintained hypermethylation of the $\mathrm{CpG}$ islands, and that high DNMT1 expression in the iPS cells was able to promote their differentiation.

\section{Discussion}

In the early stages of generation, iPS cells are generally highly efficient in their ability to be reprogrammed and possess a strong capacity for self-renewal. However, following consecutive subculturing, it was observed in this study that iPS cells began to lose these characteristics and to show decreased pluripotency as they differentiated, particularly following 40 passages. Moreover, when the iPS cells cultured on MEFs were subcultured continuously in vitro, the cells also showed a decreased expression of endogenous pluripotency stem cell markers, and the ability of the cells to differentiate into three germ layers in vivo was not as efficient as that of iPS cells cultured at earlier passages. Furthermore, assays at later passages indicated that the expression of two crucial transcriptor factors, Oct4 and Nanog, decreased rapidly during the in vitro subculture of the iPS cells on MEFs. A previous study demonstrated that the promoter regions of Oct4 and Nanog, as well as other pluripotency regulators, were methylated in somatic cells and became demethylated during reprogramming to a pluripotent state (29). The downregulation of Nanog and Oct4 expression may induce the pluripotent stem cells to differentiate (41). The effects of DNA methylation on cells include transcriptional repression by the methylation of promoter regions, and this is required in mammals for embryonic development, $\mathrm{X}$ chromosome inactivation and imprinting (42). Moreover, a previous study revealed that three human DNA methyltransferases (DNMT1, DNMT3a and 3b) were widely expressed in a coordinated fashion in the majority of normal tissues, tumors and stem cells (42). In our previous studies, we have also demonstrated that the expression of a number of growth factors (bFGF, EGF and IGF-1) and LIF by HuAECs may be crucial components by which feeder cells maintain mouse and human ESCs, as well as mouse spermatogonial stem cells, in an undifferentiated, proliferative state, capable of self-renewal $(18,21,37,39,40,43)$. With reference to these studies, we hypothesized that HuAECs as feeder layers may support the undifferentiated growth and maintain the 
pluripotency of iPS cells in long-term culture. The two main conclusions from this study are discussed in the following section.

DNMT1 was shown to induce hypermethylation of the promoters of Oct4 and Nanog, although not those of DNMT3a or $3 b$, causing the iPS cells to lose pluripotency during continuous subculture. We analyzed the expression levels of DNMT1 in iPS cells at different passages. Over time, the DNMT1 expression in these iPS cells became increasingly higher. Having confirmed that our siRNA specifically reduced endogenous DNMT1 expression in iPS cells, it was observed that the expression of pluripotent stem cell markers (Nanog and Oct4), as well as the level of AP activity, was higher in iPS cells transfected with siRNA-DNMT1 than those in iPS cells transfected with siRNA-Mock. Importantly, compared with the siRNA-Mock-transfected group, specific loci of Nanog and Oct4 were demethylated/hypomethylated in the iPS cells transfected with siRNA-DNMT1 during long-term subculture. Therefore, we concluded that DNMT1, not DNMT 3a/3b, induced the hypermethylation of Nanog and Oct4 and caused the iPS cells to lose pluripotency during long-term subculture.

A further conclusion from this study was that the use of HuAECs as feeder layers was able to maintain the pluripotency of human iPS cells during long-term subculture. It was demonstrated that the HuAEC feeder cells allowed human iPS cells to maintain a high level of AP activity. In addition, it was observed that expression levels of Nanog, Oct4 and other important stem cell markers were higher in iPS cells cultured on HuAECs compared with those cultured on MEFs during long-term subculture. Furthermore, using the sodium bisulfite and PCR assay, it was demonstrated that the $\mathrm{CpG}$ islands of the Nanog-and Oct4-specific loci were hypomethylated in iPS cells cultured on HuAECs. In addition, iPS cells cultured on $\mathrm{HuAECs}$ exhibited higher levels of histone $\mathrm{H} 3$ acetylation and lower levels of H3K 27 trimethylation at the Nanog- and Oct4-specific loci than those cultured on MEFs during subculture. Accordingly, the expression of DNMT1 in iPS cells cultured on HuAECs was lower than that in the cells cultured on MEFs. In combination, these results suggested that the HuAEC-induced epigenetic modifications at the Nanog and Oct4 loci may be a key mechanism to maintain the iPS cells in an undifferentiated, proliferative state, capable of self-renewal, through the suppression of DNMT1 expression during long-term subculture in vitro.

Importantly, the use of HuAECs, in contrast to MEFs, as feeder layers avoids contamination from heterogeneous proteins. To date, numerous studies have indicated that the in vivo proliferation and differentiation of human iPS cells is dependent on a specific microenvironment, including various cytokines, LIF and other unknown factors $(3,21,37)$. In order to maintain the self-renewal and proliferative properties and to inhibit the differentiation of iPS cells in vitro, a similar microenvironment must be provided with the essential ingredients for growth. HuAECs are temporary specialized fetal cells, derived from the placenta, which are able to maintain the pluripotency of early epiblast cells. Previous studies have indicated that HuAECs express a number of growth factors, such as LIF, EGF, bFGF, transforming growth factor (TGF)- $\alpha / \beta$ and bone morphogenetic protein (BMP)-4, as well as stem cell markers, including Nanog, Oct4 and nestin $(20,44)$. The results of our previous studies suggested that the expression of LIF by HuAECs was able to maintain mouse and human ESCs and mouse spermatogonial stem cells in an undifferentiated, proliferative state, capable of self-renewal $(19,39,40)$. Additional advantages of the human placental amnion include its low toxicity and high safety, due to the presence of few exogenous foreign proteins. Furthermore, unlike ESCs, its use is free from ethical constraints. Therefore, this present study demonstrated that the human placental amnion may be used as an abundant source of feeder cells for human iPS cultures.

\section{Acknowledgements}

This study was supported by grants from the National Natural Science Foundation of China (no. 81202811) and Shanghai Municipal Health Bureau Fund (no. 20124320) to Dr Te Liu.

\section{References}

1. Takahashi $\mathrm{K}$ and Yamanaka S: Induction of pluripotent stem cells from mouse embryonic and adult fibroblast cultures by defined factors. Cell 126: 663-676, 2006.

2. Takahashi K, Tanabe K, Ohnuki M, et al: Induction of pluripotent stem cells from adult human fibroblasts by defined factors. Cell 131: 861-872, 2007

3. Galach $\mathrm{M}$ and Utikal J: From skin to the treatment of diseases - the possibilities of iPS cell research in dermatology. Exp Dermatol 20: 523-528, 2011.

4. Maherali N, Sridharan R, Xie W, et al: Directly reprogrammed fibroblasts show global epigenetic remodeling and widespread tissue contribution. Cell Stem Cell 1: 55-70, 2007.

5. Okita K, Ichisaka $\mathrm{T}$ and Yamanaka S: Generation of germline-competent induced pluripotent stem cells. Nature 448: 313-317, 2007.

6. Hanna J, Markoulaki S, Schorderet $\mathrm{P}$, et al: Direct reprogramming of terminally differentiated mature B lymphocytes to pluripotency. Cell 133: 250-264, 2008

7. Wernig M, Meissner A, Foreman R, et al: In vitro reprogramming of fibroblasts into a pluripotent ES-cell-like state. Nature 448: 318-324, 2007.

8. Liu H, Zhu F, Yong J, et al: Generation of induced pluripotent stem cells from adult rhesus monkey fibroblasts. Cell Stem Cell 3: 587-590, 2008.

9. Yu J, Vodyanik MA, Smuga-Otto K, et al: Induced pluripotent stem cell lines derived from human somatic cells. Science 318 : 1917-1920, 2007.

10. Thier M, Munst B and Edenhofer F: Exploring refined conditions for reprogramming cells by recombinant Oct4 protein. Int J Dev Biol 54: 1713-1721, 2010.

11. Zhong B, Watts KL, Gori JL, et al: Safeguarding nonhuman primate iPS cells with suicide genes. Mol Ther 19: 1667-1675, 2011.

12. Ye Z, Zhan H, Mali P, et al: Human-induced pluripotent stem cells from blood cells of healthy donors and patients with acquired blood disorders. Blood 114: 5473-5480, 2009.

13. Ezashi T, Telugu BP, Alexenko AP, Sachdev S, Sinha S and Roberts RM: Derivation of induced pluripotent stem cells from pig somatic cells. Proc Natl Acad Sci USA 106: 10993-10998, 2009.

14. Gonzalez F, Barragan Monasterio M, Tiscornia G, et al: Generation of mouse-induced pluripotent stem cells by transient expression of a single nonviral polycistronic vector. Proc Natl Acad Sci USA 106: 8918-8922, 2009.

15. Kaji K, Norrby K, Paca A, Mileikovsky M, Mohseni P and Woltjen K: Virus-free induction of pluripotency and subsequent excision of reprogramming factors. Nature 458: 771-775, 2009.

16. Zhou H, Wu S, Joo JY, et al: Generation of induced pluripotent stem cells using recombinant proteins. Cell Stem Cell 4: 381-384, 2009.

17. YakubovE,Rechavi G, Rozenblatt S and GivolD: Reprogramming of human fibroblasts to pluripotent stem cells using mRNA of four transcription factors. Biochem Biophys Res Commun 394: 189-193, 2010. 
18. Liu T, Cheng W, Liu T, et al: Human amniotic epithelial cell feeder layers maintain mouse embryonic stem cell pluripotency via epigenetic regulation of the c-Myc promoter. Acta Biochim Biophys Sin (Shanghai) 42: 109-115, 2010.

19. Liu T, Guo L, Liu Z and Cheng W: Human amniotic epithelial cells maintain mouse spermatogonial stem cells in an undifferentiated state due to high leukemia inhibitor factor (LIF) expression. In Vitro Cell Dev Biol Anim 47: 318-326, 2011.

20. Liu T, Wu J, Huang Q, et al: Human amniotic epithelial cells ameliorate behavioral dysfunction and reduce infarct size in the rat middle cerebral artery occlusion model. Shock 29: 603-611, 2008.

21. Liu T, Cheng W, Huang Y, Huang Q, Jiang L and Guo L: Human amniotic epithelial cell feeder layers maintain human iPS cell pluripotency via inhibited endogenous microRNA-145 and increased Sox2 expression. Exp Cell Res 318: 424-434, 2012

22. Hattori N, Imao Y, Nishino K, et al: Epigenetic regulation of Nanog gene in embryonic stem and trophoblast stem cells. Genes Cells 12: 387-396, 2007.

23. Cowan CA, Atienza J, Melton DA and Eggan K: Nuclear reprogramming of somatic cells after fusion with human embryonic stem cells. Science 309: 1369-1373, 2005.

24. Freberg CT, Dahl JA, Timoskainen S and Collas P: Epigenetic reprogramming of OCT4 and NANOG regulatory regions by embryonal carcinoma cell extract. Mol Biol Cell 18: 1543-1553, 2007.

25. Simonsson S and Gurdon J: DNA demethylation is necessary for the epigenetic reprogramming of somatic cell nuclei. Nat Cell Biol 6: 984-990, 2004.

26. Tada M, Tada T, Lefebvre L, Barton SC and Surani MA Embryonic germ cells induce epigenetic reprogramming of somatic nucleus in hybrid cells. EMBO J 16: 6510-6520, 1997.

27. Bibikova M, Chudin E, Wu B, et al: Human embryonic stem cells have a unique epigenetic signature. Genome Res 16: 1075-1083, 2006.

28. Li JY, Pu MT, Hirasawa R, et al: Synergistic function of DNA methyltransferases Dnmt3a and Dnmt3b in the methylation of Oct4 and Nanog. Mol Cell Biol 27: 8748-8759, 2007.

29. Delgado-Olguin P and Recillas-Targa F: Chromatin structure of pluripotent stem cells and induced pluripotent stem cells. Brief Funct Genomics 10: 37-49, 2011.

30. Athanasiadou R, de Sousa D, Myant K, Merusi C, Stancheva I and Bird A: Targeting of de novo DNA methylation throughout the Oct-4 gene regulatory region in differentiating embryonic stem cells. PLoS One 5: e9937, 2010.

31. Tsumura A, Hayakawa T, Kumaki Y, et al: Maintenance of self-renewal ability of mouse embryonic stem cells in the absence of DNA methyltransferases Dnmt1, Dnmt3a and Dnmt3b. Genes Cells 11: 805-814, 2006

32. Fatemi M, Hermann A, Pradhan S and Jeltsch A: The activity of the murine DNA methyltransferase Dnmt1 is controlled by interaction of the catalytic domain with the N-terminal part of the enzyme leading to an allosteric activation of the enzyme after binding to methylated DNA. J Mol Biol 309: 1189-1199, 2001.
33. Sen GL, Reuter JA, Webster DE, Zhu L and Khavari PA: DNMT1 maintains progenitor function in self-renewing somatic tissue. Nature 463: 563-567, 2010.

34. Mikkelsen TS, Hanna J, Zhang X, et al: Dissecting direct reprogramming through integrative genomic analysis. Nature 454: 49-55, 2008.

35. Datta J, Ghoshal K, Sharma SM, Tajima S and Jacob ST: Biochemical fractionation reveals association of DNA methyltransferase (Dnmt) 3b with Dnmt1 and that of Dnmt 3a with a histone $\mathrm{H} 3$ methyltransferase and Hdac1. J Cell Biochem 88: 855-864, 2003

36. Ohi Y, Qin H, Hong C, et al: Incomplete DNA methylation underlies a transcriptional memory of somatic cells in human iPS cells. Nat Cell Biol 13: 541-549, 2011.

37. Liu T, Zou G, Gao Y, et al: High efficiency of reprogramming $\mathrm{CD} 34^{+}$cells derived from human amniotic fluid into induced pluripotent stem cells with Oct4. Stem Cells Dev 21: 2322-2332, 2012.

38. Miyabayashi T, Teo JL, Yamamoto M, McMillan M, Nguyen C and Kahn M: Wnt/beta-catenin/CBP signaling maintains long-term murine embryonic stem cell pluripotency. Proc Natl Acad Sci USA 104: 5668-5673, 2007.

39. Lai D, Cheng W, Liu T, Jiang L, Huang Q and Liu T: Use of human amnion epithelial cells as a feeder layer to support undifferentiated growth of mouse embryonic stem cells. Cloning Stem Cells 11: 331-340, 2009

40. Lai D, Cheng W, Liu T, Jiang L, Liu T, Huang Q and Guo L: Optimization of culture conditions to support undifferentiated growth of human embryonic stem cells. Cell Reprogram 12: 305-314, 2010.

41. Guo Y, Liu S, Wang P, et al: Expression profile of embryonic stem cell-associated genes Oct4, Sox 2 and Nanog in human gliomas. Histopathology 59: 763-775, 2011.

42. Robertson KD, Uzvolgyi E, Liang G, et al: The human DNA methyltransferases (DNMTs) 1, 3a and 3b: coordinate mRNA expression in normal tissues and overexpression in tumors. Nucleic Acids Res 27: 2291-2298, 1999.

43. Liu T, Chen Q, Huang Y, Huang Q, Jiang L and Guo L: Low microRNA-199a expression in human amniotic epithelial cell feeder layers maintains human-induced pluripotent stem cell pluripotency via increased leukemia inhibitory factor expression. Acta Biochim Biophys Sin (Shanghai) 44: 197-206, 2012.

44. Miki T, Lehmann T, Cai H, Stolz DB and Strom SC: Stem cell characteristics of amniotic epithelial cells. Stem Cells 23: 1549-1559, 2005 